\title{
On the finite sum of Kronecker sets
}

\author{
By Enji Sato
}

(Received August 10, 1975)

Let $G$ be a LCA group with dual $\hat{G}$, and $E$ a compact subset of $G$. Following Rudin [5], we say that $E$ is a Kronecker set if, to each $f \in C(E)$ with $|f|=1$ and $\varepsilon>0$, there exists $\gamma \in \widehat{G}$ such that $\|f-\gamma\|_{E}<\varepsilon$. Suppose $G$ is a metrizable $I$-group, $K$ is a compact subset of $G$, and $E$ is a perfect totally disconnected, compact metric space. Then, as is well-known, there exist two Kronecker sets $K_{1}$ and $K_{2} \subset G$, both homeomorphic to $E$, such that $K_{1}+K_{2} \supset K$ (cf. [3; Lemma 3.4], [6], and [7; Lemma 7.2]).

In this note we prove two analogs to the above result. I thank Professor S. Saeki for his useful advices.

Theorem 1 (cf. [7]) Let $T=\{|z|=1\}$ be the circle group, and $T^{\infty}$ the countable cartesian product thereof. Let also $E$ be a compact metric space with a perfect subset. Then there exist two Kronecker sets $K_{1}$ and $K_{2} \subset T^{\infty}$, both homeomorphic to $E$, such that $K_{1}+K_{2}=T^{\infty}$.

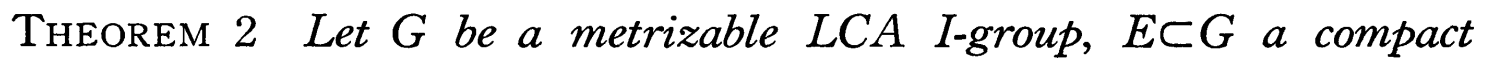
set, and $N \geq 2$ a natural number. Then there exist disjoint Kronecker sets $K_{1}, \cdots, K_{N}$, all homeomorphic to $D_{2}=\{0,1\}^{\infty}$, such that

(i) the sum $K_{1}+\cdots+K_{N}$ contains $E$, and

(ii) the union of any $N-1$ sets of the $K_{j}$ 's is a Kronecker set.

Theorem 1 is an easy consequence of the following.

Lemma 1 Let $E$ be a compact metric space, and $C\left(E ; T^{\infty}\right)$ the space of all continuous mappings from $E$ into $T^{\infty}$. Then, given $f_{0} \in C\left(E ; T^{\infty}\right)$, there exists $f \in C\left(E ; T^{\infty}\right)$ such that both $f(E)$ and $\left(f_{0}-f\right)(E)$ are Kronecker sets in $T^{\infty}$ homeomorphic to $E$.

PROOF Our proof follows Kaufman's idea in [2] (see also [1; pp. 184185]). First notice that $C\left(E ; T^{\infty}\right)$ forms a complete metric, topological group under the topology of uniform convergence. Since $E$ is a compact metric space, $C(E ; T)$ is separable. Let $\left\{g_{n}\right\}_{n=1}^{\infty}$ be a countable dense set in $C(E ; T)$. We write $N=\bigcup_{m, n} A(m, n)$, where

$$
A(m, n)=\left\{f \in C\left(E ; T^{\infty}\right):\left\|g_{m}-\chi(f)\right\|_{E} \geq 1 / n \text { for all } \chi \in \hat{T}^{\infty}\right\} .
$$

$\left(\hat{T}^{\infty}\right.$ denotes the dual group of $\left.T^{\infty}\right)$. It is obvious that every $A(m, n)$ is 
closed in $C\left(E ; T^{\infty}\right)$. Moreover we claim that $A(m, n)$ has no interior point. In fact, let $g \in C(E ; T), f \in C\left(E ; T^{\infty}\right)$, and $U$ a neighborhood of $f$ in $C\left(E ; T^{\infty}\right)$. We write $f=\left(f_{1}, f_{2}, \cdots\right)$, where $f_{n} \in C(E ; T)$ is the $n$th component of $f$. By the definition of the product topology of $T^{\infty}$, there exists a natural number $n$ such that $h \in U$, where

$$
h=\left(f_{1}, \cdots, f_{n}, g, f_{n+2}, f_{n+3}, \cdots\right) \text {. }
$$

Then, denoting by $\chi \in \hat{T}^{\infty}$ the canonical projection onto the $n+1$ th factor, we have $\chi(h)=g$ on $E$. This proves that on $A(m, n)$ has interior points and $N$ is therefore of first category in $C\left(E ; T^{\infty}\right)$.

We now prove that every $f \in C\left(E ; T^{\infty}\right) \backslash N$ is one-to-one. Choose any distinct points $a_{1}$ and $a_{2} \in E$. Since $\left\{g_{n}\right\}_{n=1}^{\infty}$ is dense in $C(E ; T)$, there exists $g_{m} \in\left\{g_{n}\right\}_{n=1}^{\infty}$ such that $g_{m}\left(a_{1}\right) \neq g_{m}\left(a_{2}\right)$. Since $f \notin N$, we can find $\chi \in \hat{T}^{\infty}$ so that

Then

$$
\left\|g_{m}-\chi(f)\right\|_{E}<3^{-1}\left|g_{m}\left(a_{1}\right)-g_{m}\left(a_{2}\right)\right|
$$

$$
\begin{aligned}
\left|g_{m}\left(a_{1}\right)-g_{m}\left(a_{2}\right)\right| & \leq 2\left\|g_{m}-\chi(f)\right\|_{E}+\left|\chi\left(f\left(a_{1}\right)\right)-\chi\left(f\left(a_{2}\right)\right)\right| \\
& \leq(2 / 3)\left|g_{m}\left(a_{1}\right)-g_{m}\left(a_{2}\right)\right|+\left|\chi\left(f\left(a_{1}\right)\right)-\chi\left(f\left(a_{2}\right)\right)\right|
\end{aligned}
$$

which confirms the one-to-oneness of $f$. Hence we obtain that $f(E)$ is homeomorphic to $E$. Finally it is easy to see that $f(E)$ is a Kronecker set whenever $f \notin N$ by the definition of $N$. Since $C\left(E ; T^{\infty}\right)$ is a complete topological group, $C\left(E ; T^{\infty}\right) \backslash\left(\left(f_{0}-N\right) \cup N\right)$ is non-empty and every element of this set has the required property.

Proof of THEOREM 1

If we construct a continuous mapping from $E$ onto $T^{\infty}$, we shall have Theorem 1 by an application of Lemma 1 . Since $E$ is a compact metric space which contains a perfect subset, it contains a compact subset homeomorphic to $D_{2}$. Since $D_{2}$ is homeomorphic to the countable product space of $D_{2}$ with itself, and since $[0,1]$ is a continuous image of $D_{2}$, the Tietze's extention theorem guarantees that $[0,1]^{\infty}$ (and hence $\left.T^{\infty}\right)$ is a continuous image of $E$. This comletes the proof.

To prove Theorem 2, we need a lemma.

LEMmA 2 Let $G$ be a LCA group, $E \subset G$ a compact set, and $n$ a natural number. Let also $V_{i}(1 \leq i \leq n)$ be open sets such that $\cup_{i=1}^{n} V_{i} \supset$ $E$. Then there exist $W_{i}(1 \leq i \leq n)$, compact neighborhoods of 0 , such that $\cup_{k=1}^{n}\left(w_{k}+V_{k}\right) \supset E$ for all $w_{k} \in W_{k}$.

PRoOF First choose compact sets $K_{i} \subset V_{i}(1 \leq i \leq n)$ so that $\cup_{i=1}^{n} K_{i} \supset$ 
E. Next take a compact neighborhood $W$ of 0 so that $K_{i}-W \subset V_{i}$ for all $1 \leq i \leq n$. Then $w_{i} \in W$ for $1 \leq i \leq n$ imply

as was required.

$$
\cup_{i=1}^{n}\left(w_{i}+V_{i}\right) \supset \bigcup_{i=1}^{n} K_{i} \supset E,
$$

ProOF OF THEOREM 2

To make the proof simple, we shall only prove Theorem 2 for $N=3$. Our proof is similar to that of Lemma 3.4 of [3].

For $r=1,2, \cdots$, we construct a finite collection $\mathscr{K}_{r}$ of distinct compact sets in $G$ with non-empty interior. First choose any compact sets $K_{1}, K_{2}$, $K_{3} \subset G$ so that

$$
\text { int } K_{1}+\text { int } K_{2}+\text { int } K_{3} \supset E \text {, }
$$

and put $\mathscr{K}_{r}=\left\{K_{r}\right\}$ for $r=1,2,3$. Suppose that $\mathscr{K}_{n}=\left\{K_{n j}\right\}_{j=1}^{p(n)}$ are constructed for all $1 \leq n \leq r+2$ and some $r \geq 1$, and that

$$
\text { int } K_{r}+\text { int } K_{r+1}+\text { int } K_{r+2} \supset E \text {, }
$$

where $K_{n}=\bigcup_{j=1}^{p(n)} K_{n j}$ for all $n$. Since $E$ is compact, there exist distinct points $x_{m} \in$ int $K_{r}$ and $x_{m}^{\prime} \in$ int $K_{r+1}(1 \leq m \leq n(r))$ such that $\bigcup_{m=1}^{n(r)}\left(x_{m}+x_{m}^{\prime}+\right.$ int $\left.K_{r+2}\right) \supset E$. There is no loss of generality in assuming that all the $K_{r j}$ (resp. $\left.K_{(r+1) \jmath}\right)$ contain at least two $x_{m}$ 's $\left(\right.$ resp. $x_{m}^{\prime}$ 's). Applying Lemma 2, we obtain disjoint compact neighborhoods $W_{r 1}, \cdots, W_{r n(r)} \subset$ int $K_{r}$ and $W_{r 1}^{\prime}, \cdots$, $W_{r n(r)}^{\prime} \subset \operatorname{int} K_{r+1}$ of these points such that

$$
\bigcup_{m=1}^{n(r)}\left(w_{m}+w_{m}^{\prime}+\operatorname{int} K_{r+2}\right) \supset E
$$

for all choices of $w_{m} \in W_{r m}$ and $w_{m}^{\prime} \in W_{r m}^{\prime}$. Since $G$ is an I-group, we can find $v_{m} \in$ int $W_{r m}$ and $v_{m}^{\prime} \in$ int $W_{r m}^{\prime}$ so that $\left\{v_{m}, v_{m}^{\prime}: 1 \leq m \leq n(r)\right\}$ is a Kronecker set (see $[5 ; 5.2])$. Choose a finite set $F_{r}$ of $\hat{G}$ so that to any real numbers $a_{m}$ and $a_{m}^{\prime}(1 \leq m \leq n(r))$ there corresponds a $\gamma \in F_{r}$ satisfying

$$
\begin{array}{ll}
\text { (1) }\left|\exp \left(i a_{m}\right)-\gamma\left(v_{m}\right)\right|<1 / r & (1 \leq m \leq n(r)) \\
(1)^{\prime}\left|\exp \left(i a_{m}^{\prime}\right)-\gamma\left(v_{m}\right)\right|<1 / r & (1 \leq m \leq n(r))
\end{array}
$$

Since $F_{r}$ is a finite set, there exist disjoint compact neighborhoods $K_{(r+3) m}$ of $v_{m}$ and $K_{(r+4) m}$ of $v_{m}^{\prime}$ such that $1 \leq m \leq n(r)$ imply

$$
\begin{aligned}
& \text { (2) }\left|\gamma(x)-\gamma\left(v_{m}\right)\right|<1 / r \quad\left(x \in K_{(r+3) m} \text { and } \gamma \in F_{r}\right) \\
& (2)^{\prime}\left|\gamma(x)-\gamma\left(v_{m}^{\prime}\right)\right|<1 / r \quad\left(x \in K_{(r+4) m} \text { and } \gamma \in F_{r}\right) \\
& \text { (3) } \operatorname{diam} K_{(r+3) m}<1 / r \text { and } K_{(r+3) m} \subset W_{r m} \\
& \text { (3) diam } K_{(r+4) m}<1 / r \text { and } K_{(r+4) m} \subset W_{r m}^{\prime} .
\end{aligned}
$$


Finally we define $\mathscr{K}_{r+k}=\left\{K_{(r+k) m}\right\}_{m=1}^{n(r)}$ for $k=3$ and 4 , which completes our inductive construction of $\left\{\mathscr{K}_{r}\right\}_{r=1}^{\infty}$.

Putting

$$
H_{k}=\bigcap_{q=0}^{\infty} \bigcup_{m} K_{(3 q+k) m} \quad(k=1,2,3),
$$

we claim that these three sets have the required properties. It is obvious that $H_{1}, H_{2}, H_{3}$ are disjoint, perfect, and totally disconnected, and that $H_{1}$ $+H_{2}+H_{3} \supset E$. Therefore we need only confirm that, say, $H_{1} \cup H_{2}$ is a Kronecker set.

Let $f \in C\left(H_{1} \cup H_{2} ; T\right)$ and $\varepsilon>0$ be given. Choose a natural number $q$ so that $1 /(q-1)<\varepsilon / 2$, and set $r=3 q-2$. Since $f$ is uniformly continuous, we can demand that there are real numbers $a_{m}$ and $a_{m}^{\prime}(1 \leq m \leq n(r))$ satisfying

$$
\begin{array}{ll}
\text { (4) }\left|f(x)-\exp \left(i a_{m}\right)\right|<\varepsilon / 2 & \left(x \in H_{1} \cap K_{(r+3) m} \text { and } 1 \leq m \leq n(r)\right) \\
(4)^{\prime}\left|f(x)-\exp \left(i a_{m}^{\prime}\right)\right|<\varepsilon / 2 & \left(x \in H_{2} \cap K_{(r+4) m} \text { and } 1 \leq m \leq n(r)\right) .
\end{array}
$$

(Notice $\bigcup_{m=1}^{n(r)} K_{(r+3) m}=\bigcup_{m=1}^{n(r)} K_{(3 q+1) m} \supset H_{1}$ and similarly for $H_{2}$.)

Choose $r \in F_{r}$ as in (1) and (1)'. We then have by (2) and (4) that

$$
\begin{aligned}
|f(x)-\gamma(x)| & \leq\left|f(x)-\exp \left(i a_{m}\right)\right|+\left|\exp \left(i a_{m}\right)-\gamma\left(v_{m}\right)\right|+\left|\gamma\left(v_{m}\right)-\gamma(x)\right| \\
& <\varepsilon / 2+1 / r+1 / r<\varepsilon / 2+1 /(q-1)<\varepsilon
\end{aligned}
$$

whenever $x \in H_{1} \cap K_{(r+3) m}$ for some $1 \leq m \leq n(r)$.

Similarly we have by $(2)^{\prime}$ and $(4)^{\prime}$

$$
|f(x)-\gamma(x)|<\varepsilon \quad\left(x \in H_{2}\right) .
$$

In other words, we have proved that $|f(x)-\gamma(x)|<\varepsilon$ for all $x \in H_{1} \cup H_{2}$ and some $r \in \hat{G}$. This completes the proof.

Remark After the first draft of this note was written, Professer S. Saeki pointed out that the following variance of Kaufman's theorem [2] yields an alternative and simple proof of Theorem 2.

Let $G$ be a metrizable LCA I-group, $H$ a $\sigma$-compact independent subset thereof, and $D$ a totally disconnected compact metric space. Then quasiall $f \in C(D ; G)$ have the properties that

(i) $f$ is one-to-one,

(ii) $f(D)$ is a Kronecker set, and

(iii) $G p(f(D)) \cap G p(H)=\{0\}$.

If, in addtion, $H$ is a totally disconnected Kronecker set, then (ii) can be 
strengthened to be (ii) $f(D) \cup H$ is a Kronecker set. (cf. [6; Lemma]). Theorem 2 follows from an inductive application of this result. We omit the details.

\section{References}

[1] Y. KAtZNELSON: An introduction to harmonic analysis. John Wiley \& Sons, New York, 1968.

[2] R. KAUfman: A functional method for linear sets. Israel J. Math, 5 (1967), 185-187.

[3] T. W. KöRNER: Some results on Kronecker, Dirichlet and Helson sets I. Ann Inst. Fourier (Grenoble), 20 (1970), 219-326.

[4] L. A. LINDAHL and F. Poulsen, (Editors): Thin sets in harmonic analysis. New York, Marcel Dekker (1971).

[5] W. RUDIN : Fourier analysis on groups. New York 1962.

[6] S. SAEKI: On the sum of two Kronecker sets. to appera in Illinois J. Math.

[7] N. Th. Varopoulos: Groups of continuous functions in harmonic analysis. Acta Math 125 (1970), 109-154.

Department of Mathematics

Tokyo Metropolitan University

Setagaya, Tokyo, Japan
Department of Mathematics

Yamagata University 